\title{
Effects of a Solvent on the Extraction of Europium(III) with 4-Isopropyltropolone
}

\author{
Junji NORO \\ Research Department, NISSAN ARC, LTD., Natsushima, Yokosuka, Kanagawa 237, Japan
}

Keywords Solvent extraction, 4-isopropyltropolone, europium(III), polynuclear chelate complex

During the course of conducting a series of studies concerning the solvent extraction of several metal ions by 2-hydroxy-4-(1-methylethyl)-2,4,6-cycloheptatrien1-one (4-isopropyltropolone; Hipt), it was found that dinuclear chelate complexes of several metal ions were extracted. ${ }^{1-3}$ Since only chloroform ${ }^{1,2}$ or only toluene ${ }^{3}$ was used as the solvent in those studies, the effects of the solvent on the extraction and formation of dinuclear chelate complexes of these metal ions were not investigated. However, it has been reported that the formation of polynuclear species with several extractants, such as carboxylic acids, was quite different for different diluents. ${ }^{4-6}$ It is thus assumed that such a diluent effect should also be found regarding the formation of dinuclear chelate complexes with 4-isopropyltropolone. In the present work, the effects of the diluent on the extraction and formation of dinuclear chelate complexes of europium(III) with 4-isopropyltropolone were studied when the solvent used was carbon tetrachloride, toluene, or 4-methyl-2-pentanone (MIBK). The results were compared with those obtained when the diluent was chloroform, as described in a previous paper. ${ }^{1}$

\section{Experimental}

All of the reagents and experimental procedures were similar to those used in previous studies ${ }^{1,2}$, except that carbon tetrachloride, toluene, and MIBK were used as the diluent.

\section{Results and Discussion}

Since a statistical treatment of the experimental data was carried out in a manner similar to that described in previous papers ${ }^{1,2}$, only the main equations are given here. In the present paper, any chemical species in the organic phase is denoted by the subscript "org" and that in the aqueous phase is given without any subscript. The 4-isopropyltropolone is denoted by HA. The distribution ratio of metal ions, $D=[\mathrm{Eu}(\mathrm{III})]_{\text {org,total }} /[\mathrm{Eu}(\mathrm{III})]_{\text {aq,total }}$, can be written as:

$$
\begin{aligned}
D & =\sum \sum j\left[\mathrm{Eu}_{j} \mathrm{~A}_{3 j} \cdot a \mathrm{HA}\right]_{\text {org }} / \sum\left[\mathrm{EuA}_{p}{ }^{3-p}\right] \\
& =\sum \sum j K_{\mathrm{exj} a}\left[\mathrm{Eu}^{3+}\right]^{(j-1)}\left[\mathrm{A}^{-}\right]^{3 j}[\mathrm{HA}]_{\text {org }} a / \sum \beta_{p}\left[\mathrm{~A}^{-}\right]^{p},
\end{aligned}
$$

where " $p$ " is zero or one in the present study and " $j$ " is the number of metal ions in the extracted species, which is either one or two. The constants, $K_{\text {exja }}$ and $\beta_{p}$, are defined as:

$$
\begin{aligned}
& K_{\mathrm{exj} a}=\left[\mathrm{Eu}_{j} \mathrm{~A}_{3 j} \cdot a \mathrm{HA}\right]_{\mathrm{org}} /\left[\mathrm{Eu}^{3+}\right]^{j}\left[\mathrm{~A}^{-}\right]^{3 j}[\mathrm{HA}]_{\mathrm{org}}{ }^{a}, \\
& \beta_{p}=\left[\mathrm{EuA}_{p}^{3-p}\right] /\left[\mathrm{Eu}^{3+}\right]\left[\mathrm{A}^{-}\right]^{p} .
\end{aligned}
$$

The total metal concentration in the organic phase can be written from Eq.(1) as:

$$
\begin{aligned}
{[\mathrm{Eu}(\mathrm{III})]_{\text {org,total }}=} & \sum \sum j\left[\mathrm{Eu}_{j}(\mathrm{ipt})_{3 j} \cdot a \mathrm{Hipt}\right]_{\text {org }} \\
& =\sum \sum j K_{\text {ex } j a}\left[\mathrm{Eu}^{3+}\right]^{j}\left[\mathrm{ipt}^{-}\right]^{3 j}[\mathrm{Hipt}]_{\text {org }}{ }^{a} . \\
& =\sum \sum j K_{\text {exja }}\left[\mathrm{Eu}^{3+}\right]_{\text {aq, total }}\left[\mathrm{ipt}^{-}\right]^{3 j}[\mathrm{Hipt}]_{\text {org }}{ }^{a} \\
& /\left(1+\beta_{1}\left[\mathrm{ipt}^{-}\right]+\cdots\right)^{j} .
\end{aligned}
$$

The concentration of 4-isopropyltropolonate anions in the aqueous phase can be calculated according to the following equation by assuming no polymerization of the reagents in the organic phase:

$$
\left[\mathrm{A}^{-}\right]=[\mathrm{HA}]_{\text {initial }} /\left\{1+\left(K_{\mathrm{d}}+1\right)\left[\mathrm{H}^{+}\right] K_{\mathrm{a}^{-1}}\right\},
$$

where $K_{\mathrm{d}}$ is the two-phase distribution constant $\left([\mathrm{HA}]_{\mathrm{org}}[\mathrm{HA}]^{-1}\right)$ and $K_{\mathrm{a}}$ is the acid-dissociation constant $\left(\left[\mathrm{H}^{+}\right]\left[\mathrm{A}^{-}\right][\mathrm{HA}]^{-1}\right)$

The two-phase distribution constant of 4-isopropyltropolone, $K_{\mathrm{d}}$, was taken from ref. 7 for carbon tetrachloride or MIBK. The values of the constant were confirmed in the same way as in a previous study. ${ }^{2}$ When the diluent was toluene, the value of this constant was determined in the same manner. The values are listed in Table 1. The value of the acid-dissociation 
Table 1 Summary of the equilibrium constants for the solvent extraction of europium(III) with 4-isopropyltropolone

\begin{tabular}{lccccc}
\hline & $K_{\mathrm{d}}$ & $\log K_{\text {ex } 11}$ & $\log K_{\text {ex } 0}$ & $\log K_{\text {ex } 21}$ & $\beta_{1}$ \\
\hline Chloroform $^{\mathrm{a}}$ & 3.37 & - & - & $53.3^{\mathrm{a}}$ & - \\
Carbon tetrachloride & 2.29 & 23.0 & - & 50.2 & 7.2 \\
Toluene & 2.41 & 23.3 & 48.7 & 50.3 & 7.2 \\
MIBK & 2.36 & 23.0 & 47.7 & $(48.1)^{\mathrm{b}}$ & 7.2 \\
\hline
\end{tabular}

a. Taken from ref. 1. b. Error is too large.

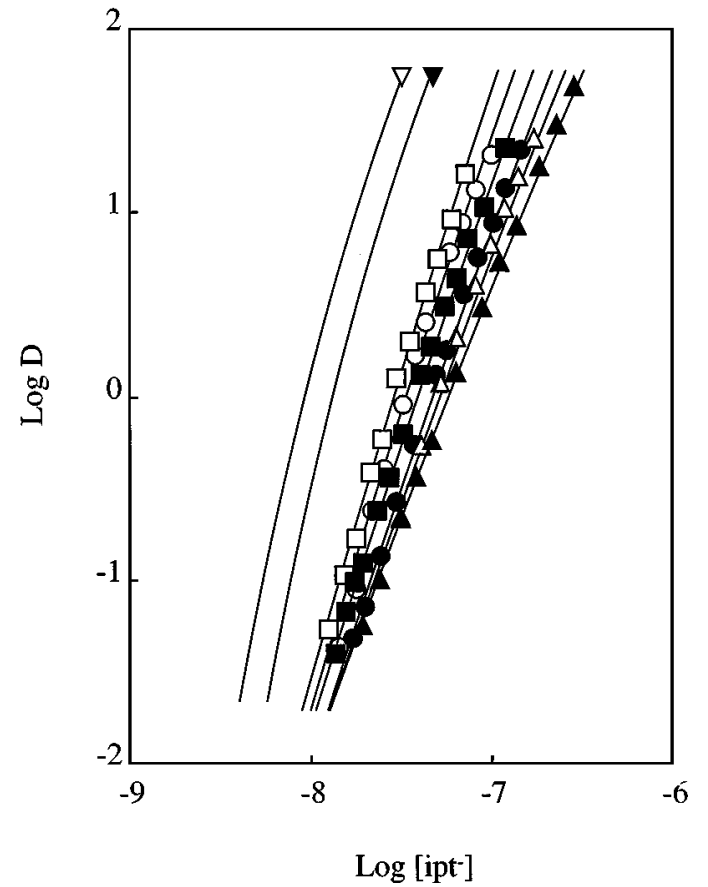

Fig. 1 Distribution ratios of europium(III) as a function of the concentration of 4-isopropyltropolonate anions in the aqueous phase. The diluent was carbon tetrachloride $(\circlearrowleft, \bullet)$, toluene $(\square, \boldsymbol{\square})$, or $\operatorname{MIBK}(\triangle, \mathbf{\Delta})$. The plots of $(\nabla, \mathbf{v})$ indicate that the diluent was chloroform taken from ref. 1. The open and closed symbols indicate that the initial metal concentration was $1 \times 10^{-4}$ and $1 \times 10^{-5} \mathrm{~mol} \mathrm{dm}^{-3}$, respectively. The initial concentration of 4-isopropyltropolone in these organic phases was $0.1 \mathrm{~mol} \mathrm{dm}^{-3}$. The solid lines were calculated with Eq.(1) by using the values of the constants in Table 1.

constant, $K_{\mathrm{a}}$, was taken from ref. 8 .

The extraction curves of $1 \times 10^{-4}$ and $1 \times 10^{-5} \mathrm{~mol} \mathrm{dm}^{-3}$ of europium(III) with $0.1 \mathrm{~mol} \mathrm{dm}^{-3}$ of 4-isopropyltropolone into carbon tetrachloride, toluene, and MIBK are given in Fig. 1. Plots for chloroform in ref. 1 are also given in Fig. 1. As can be seen from Fig. 1, the extractions of europium(III) into all four organic solvents when the initial metal concentration in the aqueous phase was $1 \times 10^{-4} \mathrm{~mol} \mathrm{dm}^{-3}$ were better than that when it was $1 \times 10^{-5} \mathrm{~mol} \mathrm{dm}^{-3}$. This suggests that europium(III) is extracted as a polynuclear species in these organic solvents, as can be seen from Eq.(1). In order to know the degree of polymerization as well as the numbers of 4-isopropyltropolonate molecules in the molecular form, Hipt, contained in the extracted

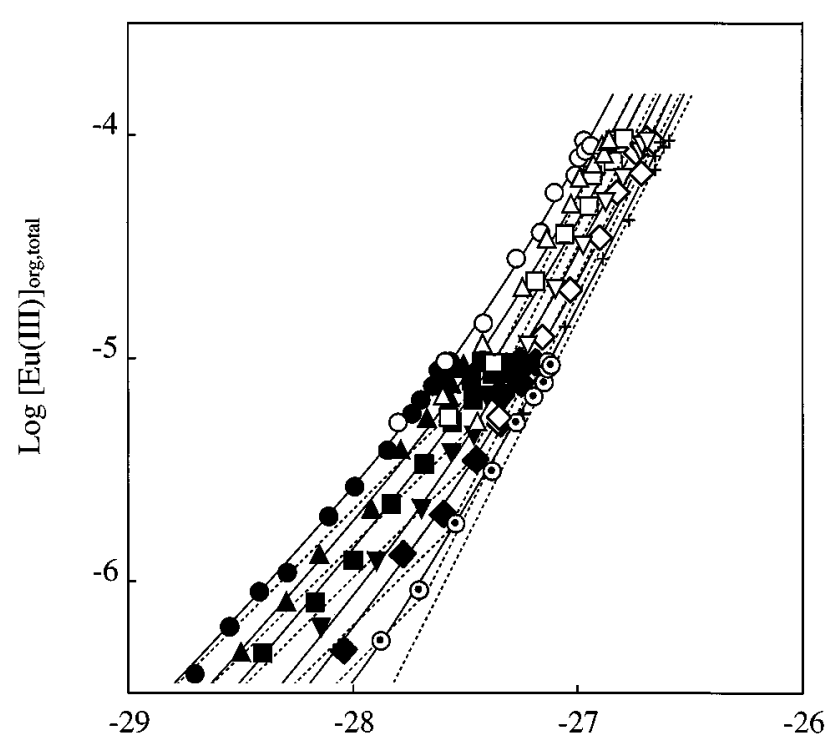

$\log \left[\mathrm{Eu}^{3+}\right]_{\text {aq,total }}\left[\mathrm{ipt}^{-}\right]^{3} /\left(1+\beta_{1}\left[\mathrm{ipt}^{-}\right]\right)$

Fig. 2 Relation between the $\log [\mathrm{Eu}(\mathrm{III})]_{\text {org,total }} v s$. $\log \left[\mathrm{Eu}^{3+}\right]_{\text {aq.total }}\left[\mathrm{ipt}^{-}\right]^{3 /}\left(1+\beta_{1}\left[\mathrm{ipt}^{-}\right]\right)$when $\beta_{1}$ is $10^{7.2}$. Each solid line was normalized to $\log Y=\log \left(K_{\text {mono }} X+K_{\mathrm{di}} X^{2}\right)$ where $Y=[\mathrm{Eu}(\mathrm{III})]_{\text {org,total }}, X=\left[\mathrm{Eu}^{3+}\right]_{\text {aq, total }}\left[\mathrm{ipt}^{-}\right]^{3} /\left(1+\beta_{1}\left[\mathrm{ipt}^{-}\right]\right)$, and $K_{\text {mono }}$ and $K_{\mathrm{di}}$ are constants whose values differ when [Hipt $]_{\text {org }}$ is different. The dotted lines are the asymptotes above the equation. The initial concentration of 4-isopropyltropolone was $0.1 \mathrm{~mol} \mathrm{dm}^{-3}(\bigcirc, \bullet), 0.07 \mathrm{~mol} \mathrm{dm}^{-3}(\triangle, \mathbf{\Delta}), 0.05 \mathrm{~mol} \mathrm{dm}^{-3}(\square$, 匹), $0.03 \mathrm{~mol} \mathrm{dm}^{-3}(\nabla, \nabla), 0.02 \mathrm{~mol} \mathrm{dm}^{-3}(\diamond, \diamond)$, and 0.01 mol dm${ }^{-3}(+, \odot)$. The initial metal concentration was $1 \times 10^{-4}$ and $1 \times 10^{-5} \mathrm{~mol} \mathrm{dm}^{-3}$, respectively.

species of europium(III), the extractions of europium(III) into these organic solvents were further examined for various 4-isopropyltropolone concentrations. As can be seen from Eq.(4), the former can be determined from the slope of the plots of $\log [\mathrm{Eu}(\mathrm{III})]_{\text {org, total }} v s . \log \left[\mathrm{Eu}^{3+}\right]_{\text {aq,total }}\left[\mathrm{ipt}^{-}\right]^{3 /}\left(1+\beta_{1}\left[\mathrm{ipt}^{-}\right]\right)$. The latter in the mononuclear and dinuclear chelate complexes can be obtained from the slopes of plots of $\log D\left[\mathrm{ipt}^{-}\right]^{-3}\left(1+\beta_{1}\left[\mathrm{ipt}^{-}\right]\right)$vs. $\log [\mathrm{Hipt}]_{\text {org }}$ and of $\log$ $D\left[\mathrm{ipt}^{-}\right]^{-6}\left(1+\beta_{1}\left[\mathrm{ipt}^{-}\right]\right) /\left[\mathrm{Eu}^{3+}\right] v s . \log [\mathrm{Hipt}]_{\text {org }}$, respectively. The details of these statistical treatments are described in refs. 1 and 2. These plots are given in Figs. 2 and 3 as an example when the diluent was toluene. In these figures, $\beta_{1}$ is $10^{7.2}$, which gives the best fit for the 


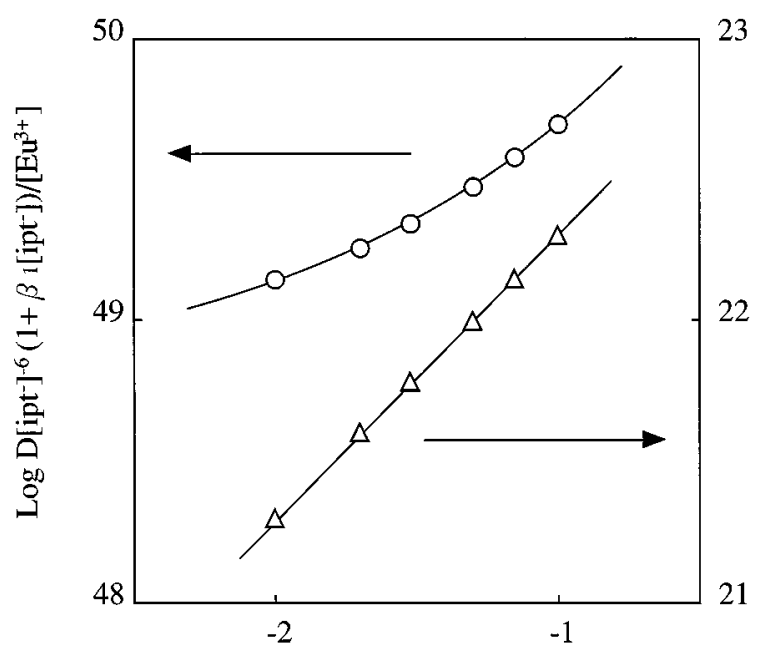

Log $[\text { Hipt }]_{\text {org }}$

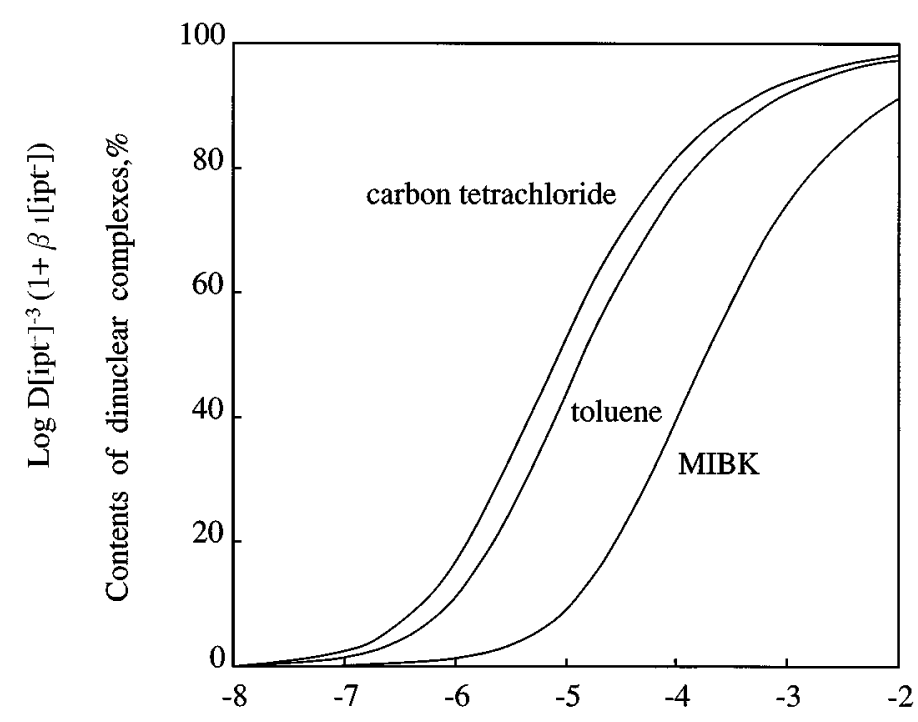

$\log [\mathrm{Eu}(\mathrm{III})]_{\text {org,total }}$

Fig. 3 Determination of the number of molecules of 4-isopropyltropolone contained in the extracted species of europium(III). Dinuclear species are indicated by $(0)$ and mononuclear species by $(\triangle)$. The solid lines were calculated with Eq.(4) using the values given in Table 1.

experimental data under these conditions. As can be seen from Fig. 2, the slopes of the plots gradually increase in the range from +1 to +2 , and the presence of both the mononuclear and dinuclear complexes was confirmed. On the other hand, as can be seen from Fig. 3 , the slope of plots of mononuclear chelate complexes of europium(III) is +1 . Thus, the number of 4-isopropyltropolonate molecules in this extracted mononuclear species should be one. However, the slope of the plots of dinuclear chelate complexes gradually increase in the range from +0 to +1 . This indicates that the extracted dinuclear species should be both $\mathrm{Eu}_{2}(\mathrm{ipt})_{6-}$ and $\mathrm{Eu}_{2}(\mathrm{ipt})_{6}$.Hipt-type chelate complexes. The chemical forms of the extracted species and the extraction constants in Eq.(2) were obtained from the experimental data by a successive approximation method. The values are listed in Table 1 . The errors of the values of the extraction constants, $\log K_{\text {exja }}$, and the formation constant in the aqueous phase, $\log \beta_{1}$, in Table 1 are smaller than \pm 0.1 , except that of $K_{\mathrm{ex} 21}$ of MIBK. The error of $K_{\mathrm{ex} 21}$ of MIBK is too large. The solid lines in Figs. 1, 2, and 3 were calculated on the basis of Eqs.(1) and (4) by using the values of the constants in Table 1.

As can be seen from Fig. 1, the extraction of europium (III) when the initial concentration of 4-isopropyltropolone and metal ions were identical was better in the following order: chloroform $>$ toluene $>$ carbon tetrachloride $>$ MIBK. This order is the same as the two-phase distribution constant of 4-isopropyltropolone, $K_{\mathrm{d}}$. It is noteworthy in Table 1 that the value of $K_{\mathrm{d}}$ of chloroform is one order larger than that of the other three solvents. Thus, it can be expected that the liquid-liquid distribution constants of 4-isopropyltropolonate chelate complexes

of europium(III) $\left(K_{\mathrm{dm}}:\left[\mathrm{Eu}_{j} \mathrm{~A}_{3 j^{*}} a \mathrm{HA}\right]_{\mathrm{org}} /\left[\mathrm{Eu}_{j} \mathrm{~A}_{3 j^{*}} a \mathrm{HA}\right]\right)$ into chloroform should be much higher than those into the other solvents. ${ }^{9}$ Therefore, the extraction into chloroform is much better than into the other solvents.

The chemical forms of the extracted species were predominantly the $\mathrm{M}_{2}(\mathrm{ipt})_{6}$. Hipt type for chloroform, both the $\mathrm{M}(\mathrm{ipt})_{3} \cdot \mathrm{Hipt}$ and $\mathrm{M}_{2}(\mathrm{ipt})_{6} \cdot$ Hipt types for carbon tetrachloride, the $\mathrm{M}(\mathrm{ipt})_{3} \cdot \mathrm{Hipt}, \mathrm{M}_{2}(\mathrm{ipt})_{6}$, and $\mathrm{M}_{2}$ (ipt) ${ }_{6}$.Hipt types for toluene, and both the $\mathrm{M}(\mathrm{ipt})_{3} \cdot \mathrm{Hipt}$ and $\mathrm{M}_{2}(\mathrm{ipt})_{6}$ types for MIBK. Figure 4 gives the distribution curves of the dinuclear and mononuclear europium(III) chelate complexes for the total concentration of the metal 4-isopropyltropolonates in the organic phase when the initial concentration of 4isopropyltropolone was $0.1 \mathrm{~mol} \mathrm{dm}^{-3}$ and the hydrogenion concentration was $10^{-3} \mathrm{~mol} \mathrm{dm}^{-3}$. From the results in Fig. 4 and the fact that mononuclear chelate complexes were not extracted into chloroform, the degree of association of each mononuclear metal chelate complex under these conditions was as follows: chloroform $>$ carbon tetrachloride $>$ toluene $>$ MIBK. Thus, the extraction due to the formation of dinuclear chelate complexes was progressively enhanced in the same order, as can be seen from Fig. 1. However, it is generally accepted that non-polar solvents, such as carbon tetrachloride, are more favorable as a solvent for the extraction of polynuclear complexes than polar ones, such as chloroform. Although, the reason why the degree of dimerization of europium(III) chelate complexes in chloroform was higher than in carbon tetrachloride or toluene cannot be clearly explained here, it may be related to the stability of the self-adduct 
mononuclear chelate complexes. Since the stability of the self-adduct mononuclear chelate complexes in carbon tetrachloride should be higher than that in chloroform as well as that in several adduct chelate complexes of neutral ligands, such as tributylphosphate or trioctylphosphine oxide ${ }^{10}$, the exchange of Hipt molecules in the self-adduct species for the mononuclear chelate complex, $\mathrm{M}(\mathrm{ipt})_{3}$, should be more impeded in carbon tetrachloride than in chloroform. Thus, the extractability of the dinuclear chelate complexes of europium(III) should be more favorable in chloroform than in carbon tetrachloride or toluene. On the other hand, when the diluent was MIBK, the degree of dimerization was much worse than with the other three solvents, as can be seen from Fig. 4. The reason for this can be attributed to the association or solvation of the mononuclear chelate complexes with MIBK molecules, which should interfere with the association of each mononuclear chelate complex.

The author is grateful to Professor Tatsuya Sekine of Department of Applied Chemistry, Kanagawa Institute of Technology who has made valuable suggestion for the present work.

\section{References}

1. J. Noro, Anal. Sci., 14, 1099 (1998).

2. T. Sekine, I. Ninomiya, M. Tebakari and J. Noro, Bull. Chem. Soc. Jpn., 70, 1385 (1997).

3. T. Sekine, A. Abdurahman, Nguyen T. K. D. and M. Tebakai, Anal. Sci., 10, 955 (1994).

4. H. Yamada and M. Tanaka, J. Inorg. Nucl. Chem., 38, 1501 (1976).

5 H. Yamada, K. Takahashi, Y. Fujii and M. Mizuta, Bull. Chem. Soc. Jpn., 57, 2847 (1984).

6. H. Yamada, K. Yajima and H. Wada, Anal. Sci., 11, 715 (1995).

7. T. Sekine and D. Dyrssen, J. Inorg. Nucl. Chem., 26, 1727 (1964).

8. D. Dyrssen, "Extraction of Metal Ions with $\beta$ Isopropyltropolone," Trans. Roy. Inst. Technol., No. 188, Stockholm, 1962.

9. T. Sekine, R. Murai and N. Ihara, J. Inorg. Nucl. Chem., 36, 2569 (1974).

10. T. Sekine and D. Dyrssen, J. Inorg. Nucl. Chem., 29, 1475 (1967).

(Received June 16, 1999) (Accepted August 23, 1999) 